01.5

\title{
Оценка амплитуды внешнего периодического воздействия при помощи малой спайковой нейронной сети в радиофизическом эксперименте
}

\author{
() В.И. Пономаренко ${ }^{1-3}$, Д.Д. Кульминский ${ }^{1-3,}$, А.В. Андреев ${ }^{3}$, М.Д. Прохоров ${ }^{1,3}$ \\ ${ }^{1}$ Саратовский филиал Института радиотехники и электроники им. В.А. Котельникова РАН, Саратов, Россия \\ ${ }^{2}$ Саратовский национальный исследовательский государственный университет им. Н.Г. Чернышевского, Саратов, Россия \\ ${ }^{3}$ Университет Иннополиса, Иннополис, Россия \\ ฯ E-mail: kulminskydd@gmail.com
}

Поступило в Редакцию 25 августа 2020г.

В окончательной редакции 25 августа 2020 г.

Принято к публикации 2 ноября 2020 г.

Предложен метод, позволяющий с помощью малой сети, состоящей из осцилляторов с нейроподобной динамикой, оценить амплитуду внешнего периодического воздействия. Метод основан на зависимости числа спайков, генерируемых сетью, от амплитуды внешнего сигнала. Эффективность метода продемонстрирована в радиофизическом эксперименте на примере сети, состоящей из генераторов ФитцХью-Нагумо.

Ключевые слова: спайковая нейронная сеть, нейроподобные осцилляторы, модель ФитцХью-Нагумо, радиофизический эксперимент.

DOI: 10.21883/PJTF.2021.04.50636.18529

Интерес к спайковым нейронным сетям обусловлен тем, что в отличие от других искусственных нейронных сетей они используют в качестве узлов биологически реалистичные модели нейронов. Наиболее известными из таких моделей нейронов являются модели Ходжкина-Хаксли, ФитцХью-Нагумо, Моррис-Лекара и Хиндмарша-Роуза [1,2]. Биологическая адекватность спайковых нейронных сетей и наличие физиологического смысла модельных параметров позволяют эффективно моделировать с помощью таких сетей процессы обработки и запоминания информации головным мозгом $[3,4]$. В настоящее время спайковые нейронные сети широко используются на практике для автоматического распознавания речи [5], обработки звуковой и зрительной информации $[6,7]$, задач обучения [8] и управления роботами [9].

Одним из основных источников информации об активности нейронов является частота следования спайков, которая представляет собой общее число спайков (импульсов), генерируемых нейронами сети в единицу времени. Известно, что внешнее периодическое воздействие, подаваемое на нейронную сеть, способно привести к изменению частоты следования спайков и, следовательно, повлиять на процесс обработки информации нейронами [10-13]. Важной задачей современной нейрофизиологии и робототехники является оценка параметров сенсорной информации, принимаемой в качестве внешнего воздействия. В настоящей работе предложен и реализован в радиофизическом эксперименте способ оценки амплитуды внешнего периодического воздействия с помощью спайковой нейронной сети. Такая сеть может быть использована в качестве входного слоя нейронной сети системы управления движением роботов в изменяющихся условиях внешней среды.

В качестве базового элемента спайковой нейронной сети мы выбрали нейроподобный осциллятор, описываемый уравнениями ФитцХью-Нагумо [14], связали осцилляторы редкими связями и подали на каждый из них одинаковое внешнее гармоническое воздействие. Динамика осцилляторов описывается при этом модельными уравнениями следующего вида:

$$
\begin{aligned}
\varepsilon \dot{u}_{i}(t)= & u_{i}(t)-\frac{u_{i}^{3}(t)}{3}-v_{i}(t)+\sum_{j=1(j \neq i)}^{N} k_{i, j}\left(u_{j}(t)-u_{i}(t)\right) \\
& +B \sin (2 \pi f t), \quad \dot{v}_{i}(t)=u_{i}(t)+a_{i}
\end{aligned}
$$

где $i=1, \ldots, N-$ номер осциллятора, $N$ - количество осцилляторов, $u_{i}(t)$ описывает динамику мембранного потенциала нейрона, $v_{i}(t)$ отвечает за восстановление потенциала покоя мембраны, $\varepsilon$ - параметр соотношения временны́х масштабов, являющийся обычно малой величиной, $a_{i}$ - пороговый параметр, $k_{i, j}$ - коэффициент связи, действующей со стороны $j$-го элемента на $i$-й, $B$ и $f$ - амплитуда и частота внешнего гармонического воздействия соответственно. В отсутствие связей и внешнего воздействия осциллятор (1) при $a_{i}>1$ находится в локально устойчивом состоянии равновесия и является возбудимым, а при $a_{i}<1$ демонстрирует периодические автоколебания, возникающие в результате бифуркации Хопфа при $a_{i}=1$ [14]. Колебания переменной $u_{i}(t)$ при $a_{i}<1$ качественно воспроизводят спайки, генерируемые настоящими нейронами.

Для экспериментального исследования системы (1) нами была построена оригинальная радиотехническая 


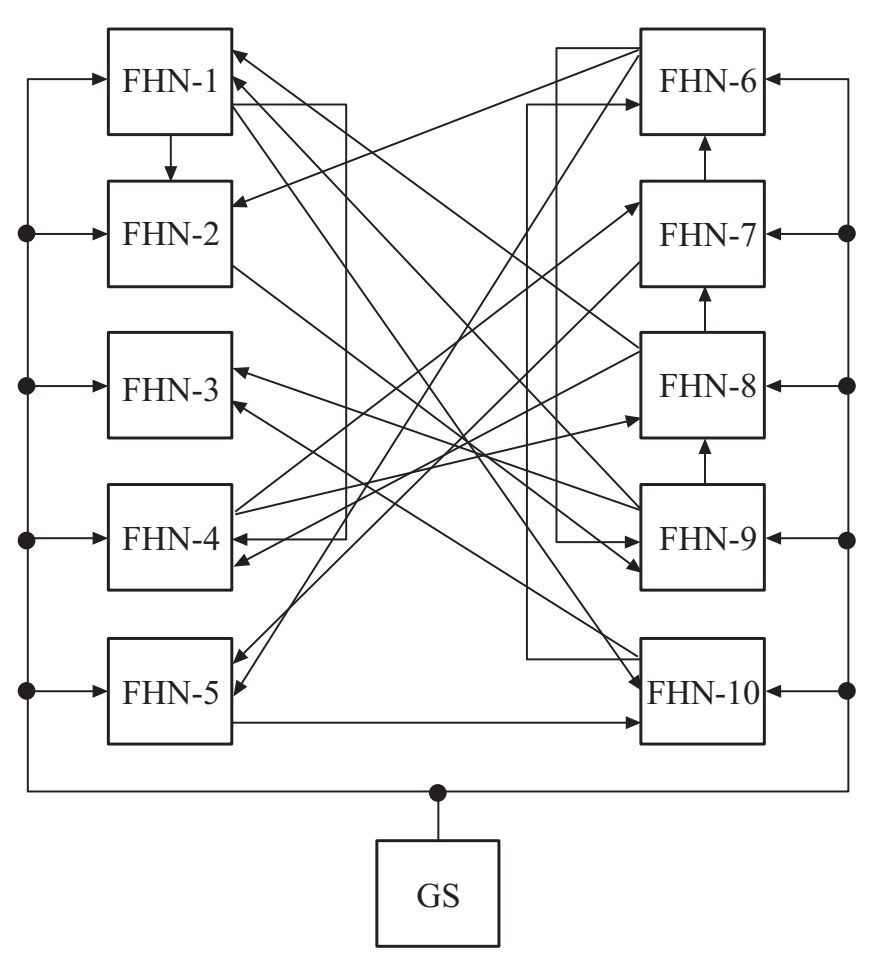

Рис. 1. Блок-схема экспериментальной установки. FHN генераторы ФитцХью-Нагумо, GS - генератор внешнего гармонического сигнала. Стрелками показаны связи между генераторами ФитцХью-Нагумо и внешнее воздействие, подаваемое на генераторы.

установка, блок-схема которой представлена на рис. 1 . Для осуществления связей между десятью генераторами ФитцХью-Нагумо, принципиальные схемы которых мы здесь не приводим, был использован предложенный нами недавно подход, в основе которого лежит программный способ формирования сигналов, отвечающих за связь между генераторами [15]. Такой подход позволяет задать произвольную архитектуру и тип связей между генераторами.

Все генераторы ФитцХью-Нагумо были выбраны неидентичными и отличались величиной параметра $a_{i}$, который принимал значения от $a_{1}=1.1 \mathrm{~V}$ до $a_{10}=1.325 \mathrm{~V}$ с шагом $0.025 \mathrm{~V}$. Без внешнего воздействия при таких значениях $a_{i}$ генерация спайков отсутствует. Архитектура связей была выбрана таким образом, чтобы на каждый из генераторов действовало по два других генератора, номера которых были выбраны случайно. Это означает, что в уравнении (1) из девяти коэффициентов связи $k_{i, j}$ для каждого генератора семь коэффициентов $k_{i, j}$ были равны нулю. Значения всех ненулевых коэффициентов связей в сети были выбраны одинаковыми: $k_{i, j}=0.01$, а параметр $\varepsilon=0.1 \mathrm{~ms}$. Мы исследовали режимы генерации спайков в зависимости от амплитуды и частоты внешнего воздействия.

На рис. 2 приведены экспериментальные временны́е реализации динамических переменных $u_{i}(t)$ всех генераторов исследуемой спайковой нейронной сети при по- стоянных значениях параметра внешнего гармонического воздействия $y(t)(B=0.2 \mathrm{~V}$ и $f=240 \mathrm{~Hz})$, временна́я реализация которого тоже показана на рисунке. Частота $f$ выбрана вблизи собственной частоты генераторов, на которой происходит генерация спайков. Из рис. 2 видно, что при таком воздействии первый генератор демонстрирует периодические спайки, частота следования которых совпадает с частотой внешнего гармонического сигнала. Второй генератор также демонстрирует спайковую активность, однако количество спайков немного меньше, чем у первого генератора. Колебания генераторов, имеющих номера с третьего по шестой, содержат пачки из четырех, трех и двух последовательных спайков. Во временны́х реализациях седьмого и восьмого генераторов одиночные спайки чередуются с подпороговыми колебаниями малой амплитуды, а девятый и десятый генераторы вообще не генерируют спайки (рис. 2).

При уменьшении амплитуды $B$ внешнего воздействия количество спайков, генерируемых нейроподобными осцилляторами (1), уменьшается, а при увеличении $B$ количество спайков в сети растет. Это свойство спайковой нейронной сети можно использовать для оценки амплитуды внешнего периодического воздействия, пода-

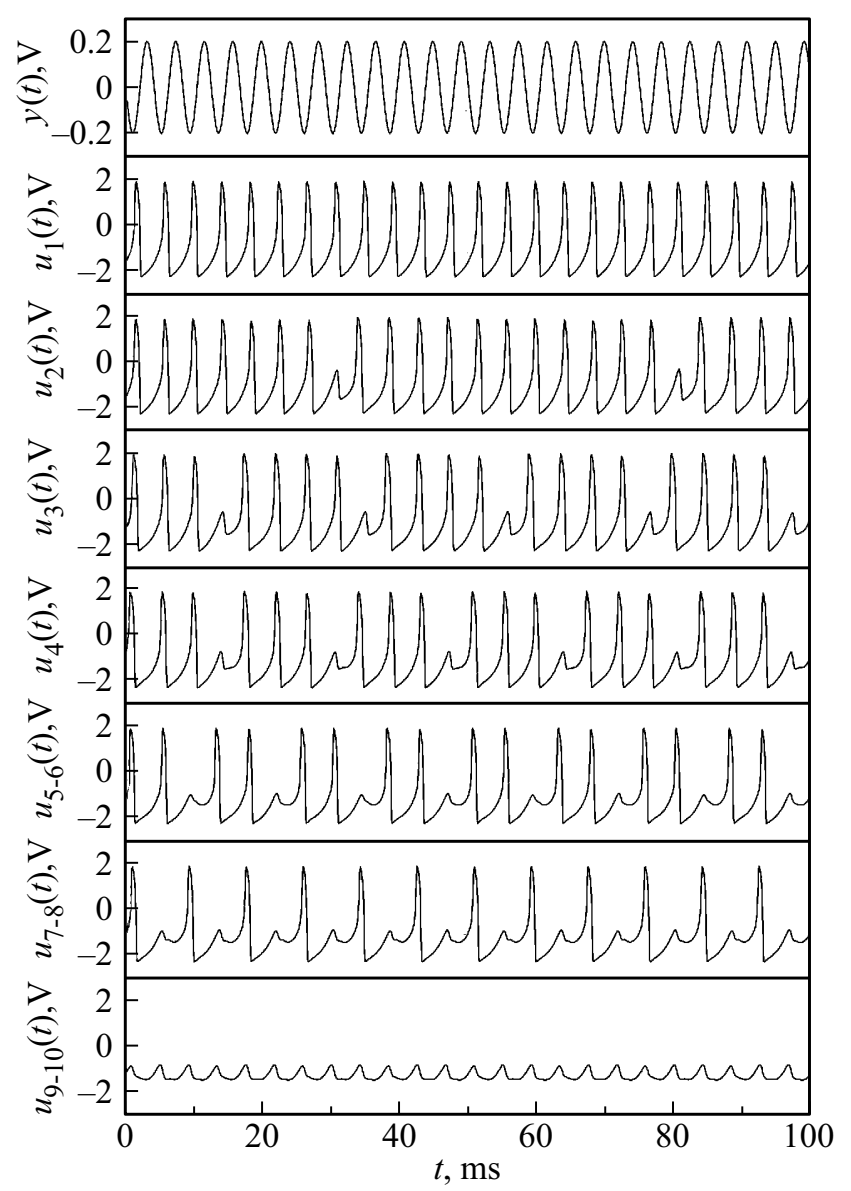

Рис. 2. Временной ряд внешнего гармонического воздействия $y(t)=B \sin (2 \pi f t)$ при $B=0.2 \mathrm{~V}, f=240 \mathrm{~Hz}$ и экспериментальные временны́е ряды динамических переменных $u_{i}(t)$ генераторов сети. 


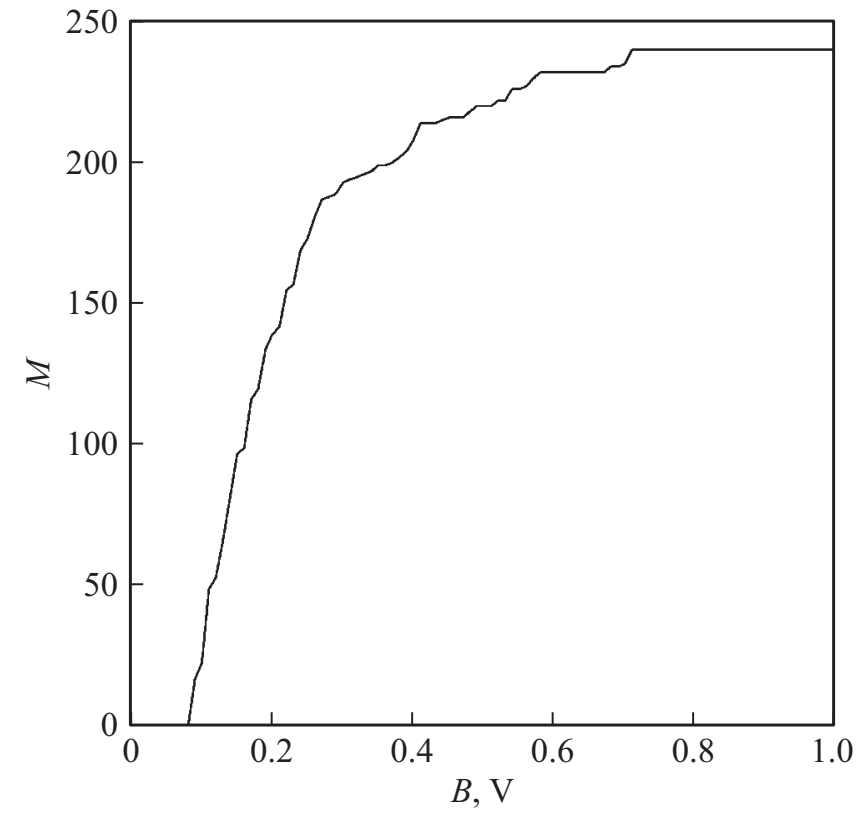

Рис. 3. Зависимость количества спайков $M$, генерируемых сетью, от амплитуды $B$ внешнего гармонического воздействия $y(t)$.

ваемого на ее элементы. Мы меняли в эксперименте амплитуду $B$ гармонического воздействия от 0 до $1 \mathrm{~V}$ с шагом $0.01 \mathrm{~V}$ и подсчитывали общее количество $M$ спайков, генерируемых всеми десятью генераторами за $100 \mathrm{~ms}$ при фиксированном значении $B$. Полученный результат представлен на рис. 3 при $f=240 \mathrm{~Hz}$. Построенный график позволяет оценить неизвестную амплитуду $B$ внешнего гармонического воздействия, подсчитав количество спайков $M$, наблюдаемых в сети. Например, если $M=139$, то $B=0.2 \mathrm{~V}$, что согласуется с рис. 2 . Увеличение числа $N$ осцилляторов спайковой нейронной сети позволяет повысить точность оценки амплитуды внешнего воздействия.

Исследования показывают, что предложенный способ оценки амплитуды периодического воздействия оказывается работоспособным в широком диапазоне частот внешнего воздействия и практически не зависит от количества и архитектуры связей в спайковой нейронной сети. Основным требованием метода является расстройка параметров осцилляторов, отвечающих за возбуждение спайковой активности. Коэффициенты связи $k_{i, j}$ между осцилляторами должны быть малы. При больших $k_{i j}$ точность метода снижается, так как зависимость $M(B)$ на рис. 3 становится слишком крутой, быстро переходя от минимального к максимальному значению $M$. Результаты, полученные в описанном радиофизическом эксперименте, хорошо согласуются с результатами проведенных нами численных исследований системы (1). Отметим, что предложенный метод позволяет оценить с помощью спайковой нейронной сети амплитуду не толь- ко гармонического, но и импульсного периодического воздействия.

Итак, нами предложен и экспериментально реализован способ оценки амплитуды внешнего гармонического воздействия при помощи малой спайковой нейронной сети. В качестве элементов сети использованы нейроподобные радиотехнические генераторы ФитцХью-Нагумо, расстроенные по пороговому параметру, отвечающему за возбуждение генератора. Показано, что количество спайков, генерируемых сетью, зависит от амплитуды внешнего стимула. Полученные результаты могут быть востребованы в робототехнике при решении задач обработки информации, связанных с управлением движением роботов, и в нейрофизиологии при использовании электрической стимуляции головного мозга [11].

\section{Финансирование работы}

Работа выполнена при поддержке Российского научного фонда (грант № 17-72-30003).

\section{Конфликт интересов}

Авторы заявляют, что у них нет конфликта интересов.

\section{Список литературы}

[1] M.I. Rabinovich, P. Varona, A.I. Selverston, H.D.I. Abarbanel, Rev. Mod. Phys., 78, 1213 (2006). DOI: $10.1103 /$ RevModPhys.78.1213

[2] А.С. Дмитричев, Д.В. Касаткин, В.В. Клиньшов, С.Ю. Кириллов, О.В. Масленников, Д.С. Щапин, В.И. Некоркин, Изв. вузов. Прикладная нелинейная динамика, 26 (4), 5 (2018). DOI: 10.18500/0869-6632-2018-226-4-5-58

[3] R.Q. Quiroga, S. Panzeri, Principles of neural coding (CRC Press, 2013).

[4] S.A. Lobov, A.V. Chernyshov, N.P. Krilova, M.O. Shamshin, V.B. Kazantsev, Sensors, 20, 500 (2020). DOI: $10.3390 / \mathrm{s} 20020500$

[5] D. Yu, L. Deng, Automatic speech recognition (Springer, 2016). DOI: 10.1007/978-1-4471-5779-3

[6] N.K. Kasabov, Evolving connectionist systems: the knowledge engineering approach (Springer, 2007). DOI: $10.1007 / 978-1-84628-347-5$

[7] M.S. Hossain, G. Muhammad, Information Fusion, 49, 69 (2019). DOI: 10.1016/j.inffus.2018.09.008

[8] S.A. Lobov, A.N. Mikhaylov, M. Shamshin, V.A. Makarov, V.B. Kazantsev, Front. Neurosci., 14, 88 (2020). DOI: $10.3389 /$ fnins.2020.00088

[9] Z. Bing, C. Meschede, F. Röhrbein, K. Huang, A.C. Knoll, Front. Neurorobot., 12, 35 (2018). DOI: $10.3389 /$ fnbot.2018.00035

[10] N. Novikov, B. Gutkin, Phys. Rev. E, 101, 052408 (2020). DOI: 10.1103/PhysRevE.101.052408

[11] D.M. Eidum, C.S. Henriquez, Chaos, 30, 033105 (2020). DOI: $10.1063 / 1.5126104$ 
[12] A.V. Andreev, M.V. Ivanchenko, A.N. Pisarchik, A.E. Hramov, Chaos Solit. Fract., 139, 110061 (2020). DOI: 10.1016/j.chaos.2020.110061

[13] M. Rooy, H.A. Новиков, Д.Г. Захаров, Б.С. Гуткин, Изв. вузов. Прикладная нелинейная динамика, 28 (1), 90 (2020). DOI: 10.18500/0869-6632-2020-28-1-90-97

[14] M.A. Dahlem, G. Hiller, A. Panchuk, E. Schöll, Int. J. Bifurcat. Chaos, 19, 745 (2009). DOI: 10.1142/S0218127409023111

[15] Д.Д. Кульминский, В.И. Пономаренко, И.В. Сысоев, М.Д. Прохоров, Письма в ЖТФ, 46 (4), 26 (2020).

DOI: 10.21883/PJTF.2020.04.49046.18018 [Пер. версия: $10.1134 / \mathrm{S} 1063785020020236$. 